\title{
Comparative Evaluation of the Remineralization Potential of Monofluorophosphate, Casein Phosphopeptide-Amorphous Calcium Phosphate and Calcium Sodium Phosphosilicate on Demineralized Enamel Lesions: An In Vitro Study
}

Kavita Kumar $^{1}$, Sheela Sreedharan ${ }^{2}$

1. Pediatric Dentist, Innocent Smiles Dental Clinic, Ernakulam, IND 2. Department of Pedodontics and Preventive Dentistry, Principal, Government Dental College, Alappuzha, IND

$\square$ Corresponding author: Kavita Kumar, 007.kavita@gmail.com

Disclosures can be found in Additional Information at the end of the article

\section{Abstract}

Aim: The aim of the study is to compare the remineralization potential of monofluorophosphate, casein phosphopeptide-amorphous calcium phosphate (CPP-ACP), and calcium sodium phosphosilicate on demineralized enamel lesions.

Materials and methods: Enamel sections from 30 sound human premolar crowns were prepared and sectioned into quadrants. Early enamel lesions were created in each sample by immersion in a demineralizing solution for 72 hours. Of the four sections, the first quadrant (A) was not given any surface treatment, the second quadrant (B) was treated with monofluorophosphate dentifrice, the third $(C)$ was treated with casein phosphopeptide-amorphous calcium phosphate (CPP-ACP), and the fourth (D) was treated with calcium sodium phosphosilicate while being subjected to a five-day $\mathrm{pH}$ cycling protocol. The sections were further cross-sectioned to expose the lesion depth and were then viewed under the confocal laser scanning microscope after staining with $0.1 \mathrm{mM}$ rhodamine B dye for 24 hours. The two parameters evaluated were the cross-sectional demineralized lesion area and total fluorescence.

Results: Amongst the dentifrices tested, the lowest values for lesion area and total fluorescence were recorded by calcium sodium phosphosilicate (3874.1 $\mu^{2}$ and 107282.6, respectively),

Received 05/15/2018 Review began 07/24/2018 Review ended 07/25/2018 Published 07/27/2018

\section{(c) Copyright 2018}

Kumar et al. This is an open access article distributed under the terms of the Creative Commons Attribution License CC-BY 3.0., which permits unrestricted use, distribution, and reproduction in any medium, provided the original author and source are credited. followed by casein phosphopeptide-amorphous calcium phosphate $\left(5776.6 \mu^{2}\right.$ and 129470.8$)$ and then by monofluorophosphate dentifrice (7371.2 $\mu^{2}$ and 233765.9) in increasing order. The highest values for lesion area and total fluorescence were recorded by the no treatment group

(16449.2 $\mu^{2}$ and 759743.1). One-way analysis of variance (ANOVA) showed significant variations $(p<0.01)$ between the groups and Scheffe multiple comparisons confirmed the significance $(p<0.01)$ of intergroup variations.

Conclusion: The results of this study suggest that, among the three agents tested, calcium sodium phosphosilicate is the most effective remineralizing agent followed by casein phosphopeptide-amorphous calcium phosphate. Monofluorophosphate is the least effective remineralizing agent when tested under the conditions mentioned in this study.
How to cite this article

Kumar K, Sreedharan S (July 27, 2018) Comparative Evaluation of the Remineralization Potential of Monofluorophosphate, Casein Phosphopeptide-Amorphous Calcium Phosphate and Calcium Sodium Phosphosilicate on Demineralized Enamel Lesions: An In Vitro Study. Cureus 10(7): e3059. DOI 
Categories: Miscellaneous, Quality Improvement, Healthcare Technology

Keywords: remineralization, calcium sodium phosphosilicate, monofluorophosphate, confocal laser scanning microscopy, cpp-acp

\section{Introduction}

Demineralization and remineralization occur in the mouth several times daily as a dynamic process, with the progression or reversal of dental caries being the end result. Whether a lesion will progress, stay the same, or reverse is determined by the balance between protective factors and pathological factors [1]. If the pathological factors that lead to demineralization predominate, then caries progresses. If the protective factors that lead to remineralization predominate, then caries is halted or reversed.

Though fluoride is the cornerstone of non-invasive caries management, its use predominantly leads to surface-only mineralization [2]. Surface-only remineralization does little to improve the aesthetics and structural properties of deeper lesions. Ideally, a remineralization system should supply stabilized bioavailable calcium, phosphate, and fluoride ions that favor subsurface mineral gain rather than deposition only in the surface layer.

The casein phosphopeptide-amorphous calcium phosphate technology has been developed based on the stabilizing properties of milk and salivary proteins. The casein phosphopeptides allow high concentrations of calcium, phosphate, and fluoride ions to be stabilized in solution, in a form that is bioavailable for the promotion of remineralization [3].

Calcium sodium phosphosilicate has been developed under the brand name Novamin (GlaxoSmithKline, Brentford, United Kingdom) and seems to be a promising alternative to promote the remineralization of early enamel lesions [4].

This in vitro study was, hence, carried out to compare the remineralization potential of monofluorophosphate, casein phosphopeptide-amorphous calcium phosphate, and calcium sodium phosphosilicate.

\section{Materials And Methods}

\section{Collection and storage of teeth}

Thirty maxillary first and second premolars extracted for orthodontic treatment with intact surfaces and no evidence of caries, hypoplasia, or restorations were used for the preparation of the specimens. All the teeth were stored in $0.1 \%$ thymol (w/v) solution until sterilization. They were cleaned of surface debris and were sterilized by autoclaving at 15 psi pressure at $121^{\circ} \mathrm{C}$ for 40 minutes [5].

\section{Sectioning and mounting}

Each tooth was sectioned into quadrants along both mesiodistal and buccolingual planes and subjected to mechanical polishing with successive 120, 400, 600, and 1200 grit paper to expose the virgin enamel in the deeper layers [6] (Figure $)$. 


\section{Cureus}

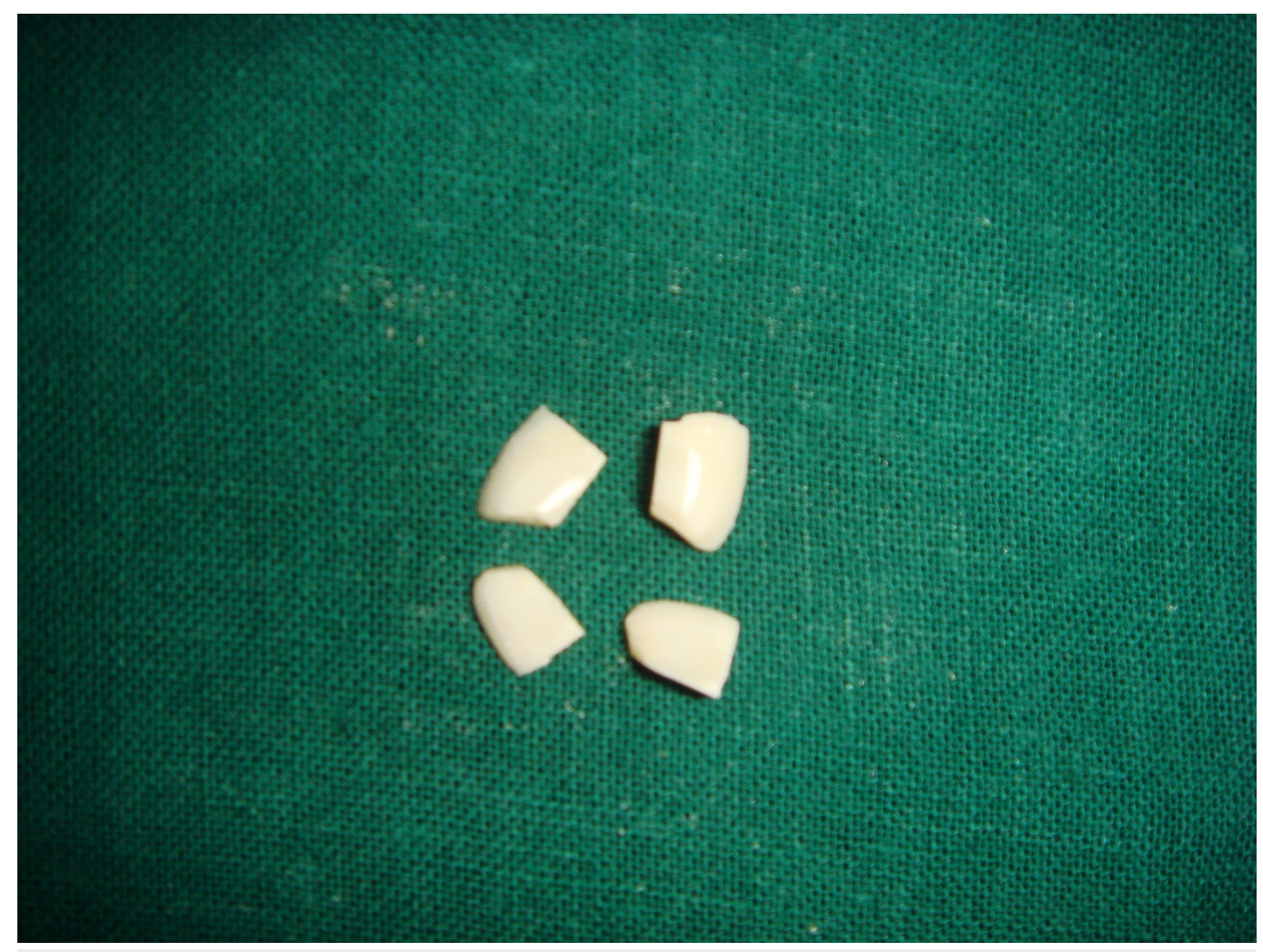

FIGURE 1: Sectioned quadrants of tooth

Sections were mounted individually in self-cure acrylic resin with the enamel surface facing the top (Figure 2).

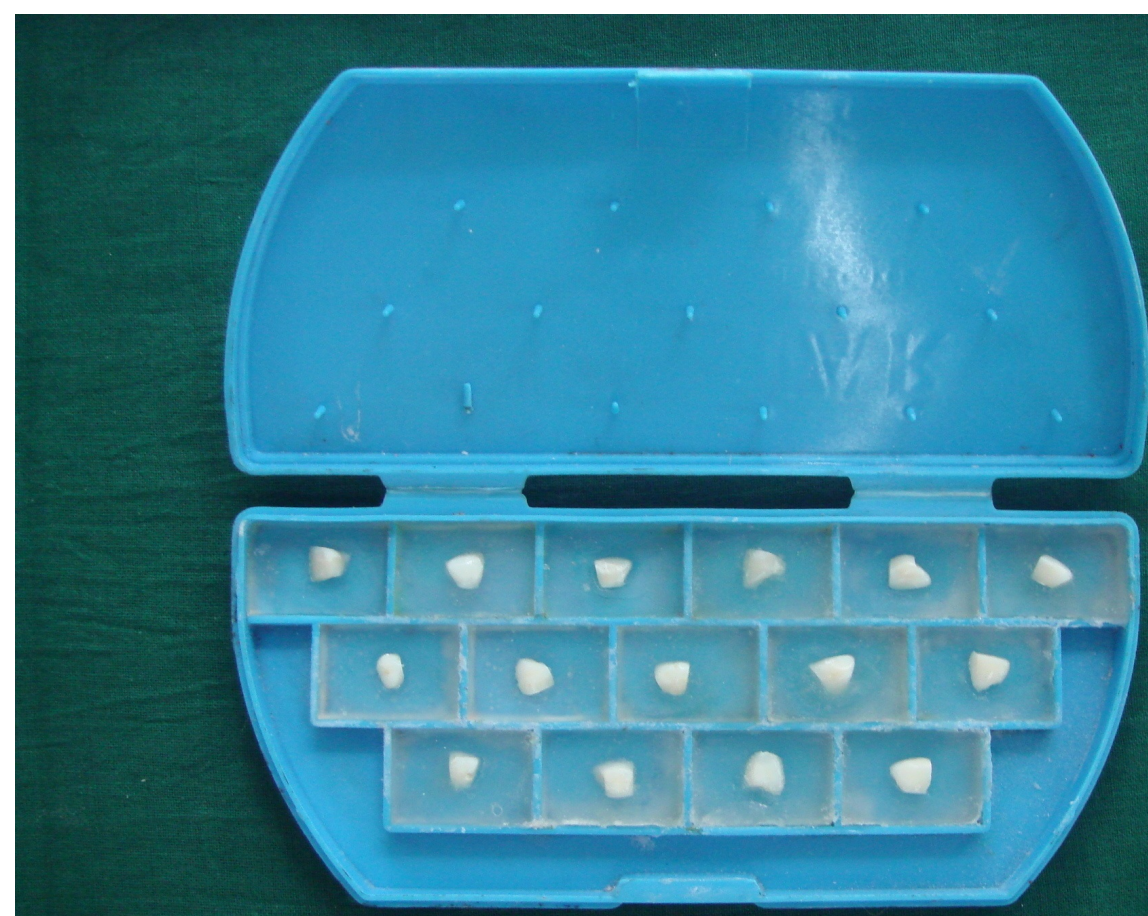




\section{Cureus}

\section{FIGURE 2: Mounting of the sections in self-cure acrylic resin}

A $3 \mathrm{~mm} \times 3 \mathrm{~mm}$ treatment window was then opened on each tooth section by applying acid-resistant nail varnish (Revlon, New York, USA) [7] on all other areas of the acrylic block except for the area designated as the treatment window. They were then labeled according to the treatment protocol (Figure 3).

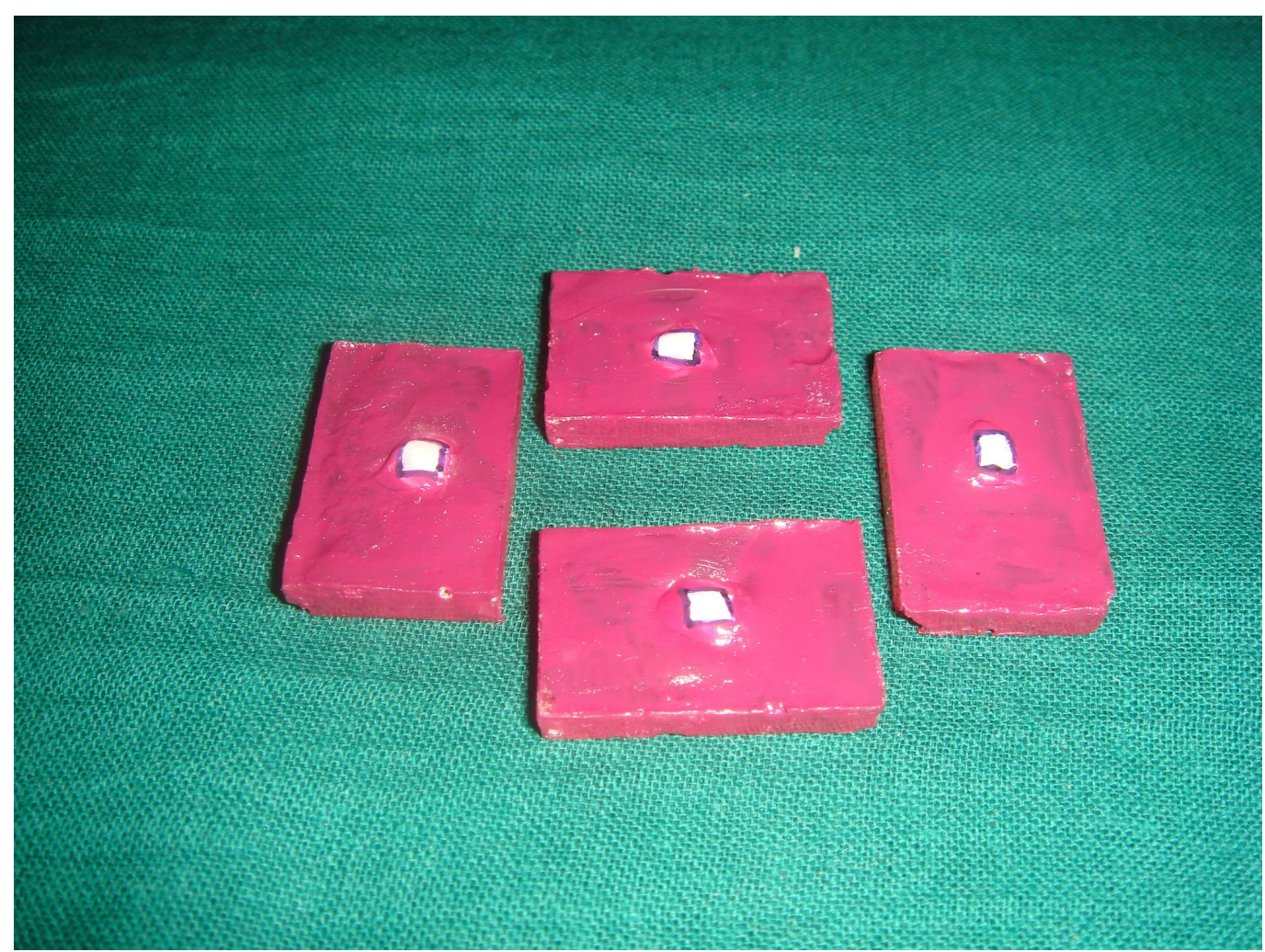

FIGURE 3: Sample after the application of nail varnish

\section{Lesion formation}

The specimens were kept in the demineralization solution $\left(2.2 \mathrm{mM} \mathrm{CaCl}_{2} 2.2 \mathrm{mM} \mathrm{KH}_{2} \mathrm{PO}_{4}, 0.05\right.$ $\mathrm{M}$ lactic acid, 0.5 ppm fluoride; $\mathrm{pH}$ adjusted to 4.5 using $50 \% \mathrm{NaOH}$ solution) for 72 hours, at $37^{\circ} \mathrm{C}$. After 72 hours of demineralization, the specimens were washed thoroughly in distilled water to remove any traces of the solution [8].

\section{pH-cycling treatment protocol}

The tooth specimens were subjected to a $\mathrm{pH}$-cycling protocol, including alternate immersion in the demineralization solution (detailed above) and the remineralization solution (3.90 mM $\mathrm{Na}_{3} \mathrm{PO}_{4}, 17.98 \mathrm{mM} \mathrm{KCl}, 0.08 \mathrm{mM} \mathrm{MgCl}_{2}, 3.27 \mathrm{mM} \mathrm{NaHCO}_{3}, 4.29 \mathrm{mM} \mathrm{NaCl}, 1.10 \mathrm{mM} \mathrm{CaCl}_{2}$ and $0.50 \mathrm{mM} \mathrm{H}_{2} \mathrm{SO}_{4}$; $\mathrm{pH}$ adjusted to $7.2 \mathrm{using} 85 \%$ lactic acid at $37^{\circ} \mathrm{C}$ with in-between dentifrice treatment done at room temperature [9]. The dentifrices used were monofluorophosphate dentifrice (Colgate Regular; Colgate-Palmolive (India) Ltd.) containing 1000 ppm fluoride, casein phosphopeptide-amorphous calcium phosphate (GC Tooth Mousse, GC Corporation, Tokyo, Japan) containing 10\% w/w CPP-ACP and calcium sodium phosphosilicate dentifrice 


\section{Cureus}

(SHY NM, Group Pharmaceuticals Ltd., Mumbai, India) containing 5\% w/w Novamin for a period of five days. The first quadrant was not given any dentifrice treatment and was designated as group A (Figure 4),

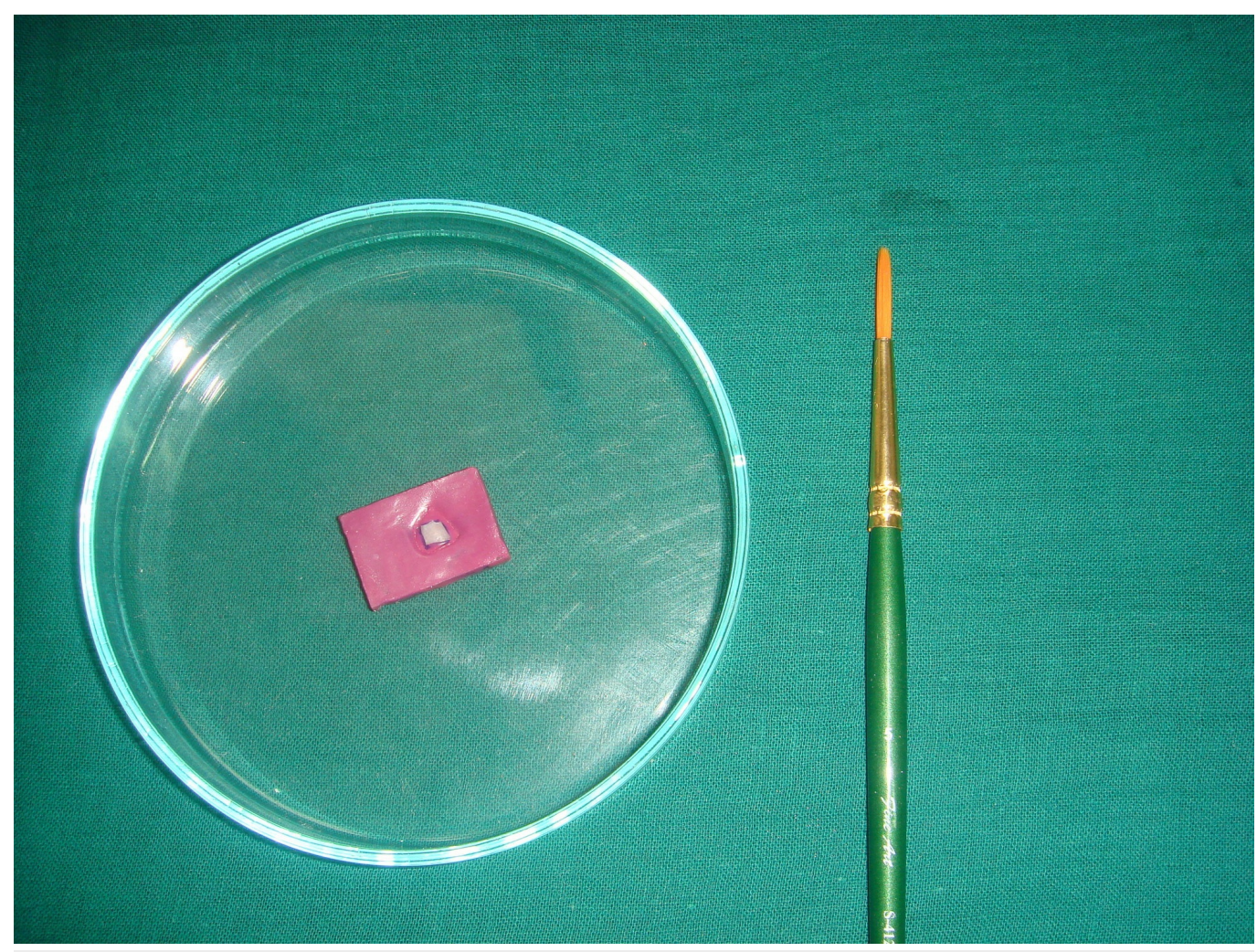

FIGURE 4: Group A: no treatment

The second quadrant was treated with monofluorophosphate dentifrice and designated as group B (Figure 5). 


\section{Cureus}

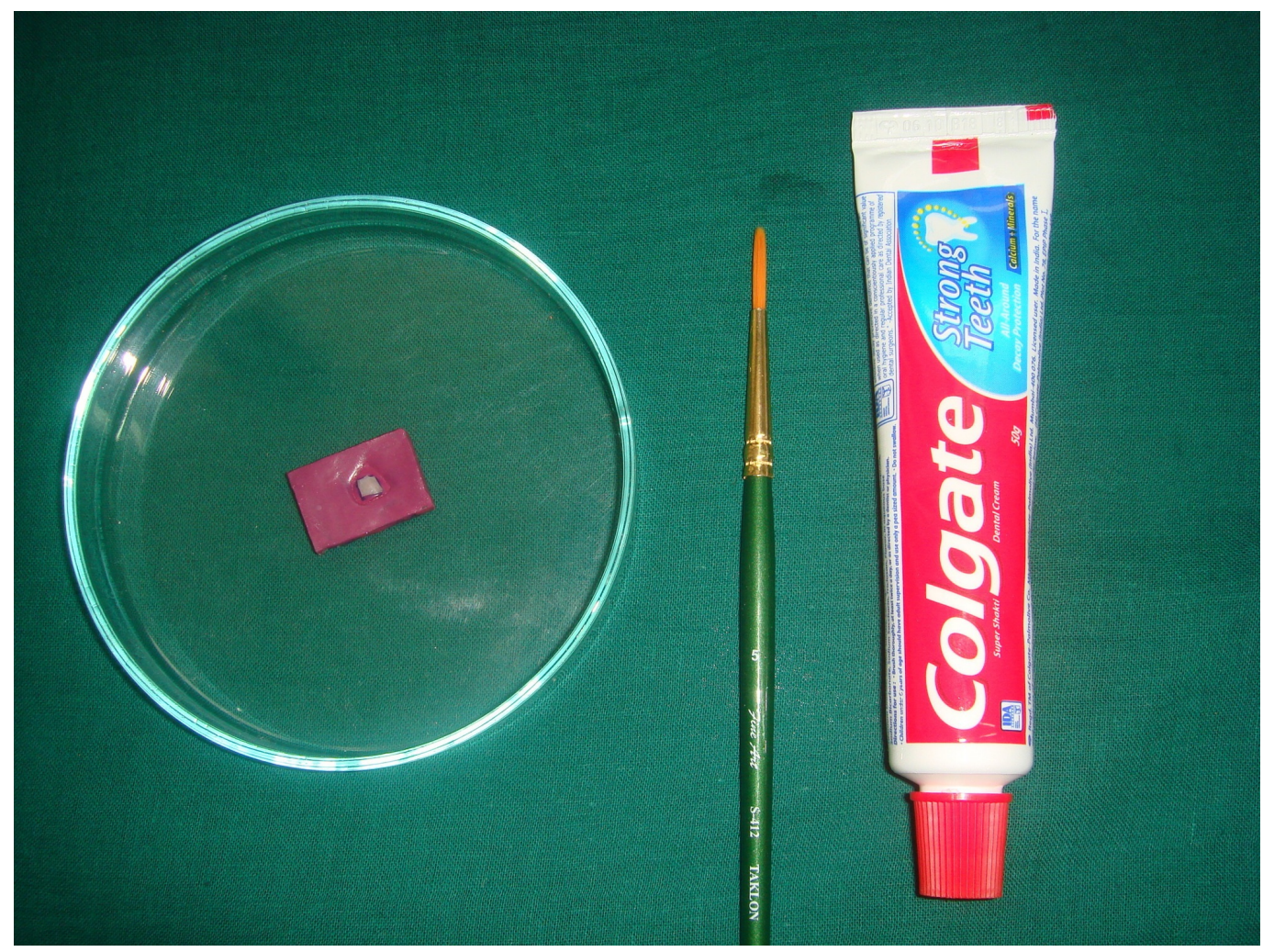

FIGURE 5: Group B: monofluorophosphate treatment

The third quadrant was treated with CPP-ACP and was designated as group C (Figure 6).

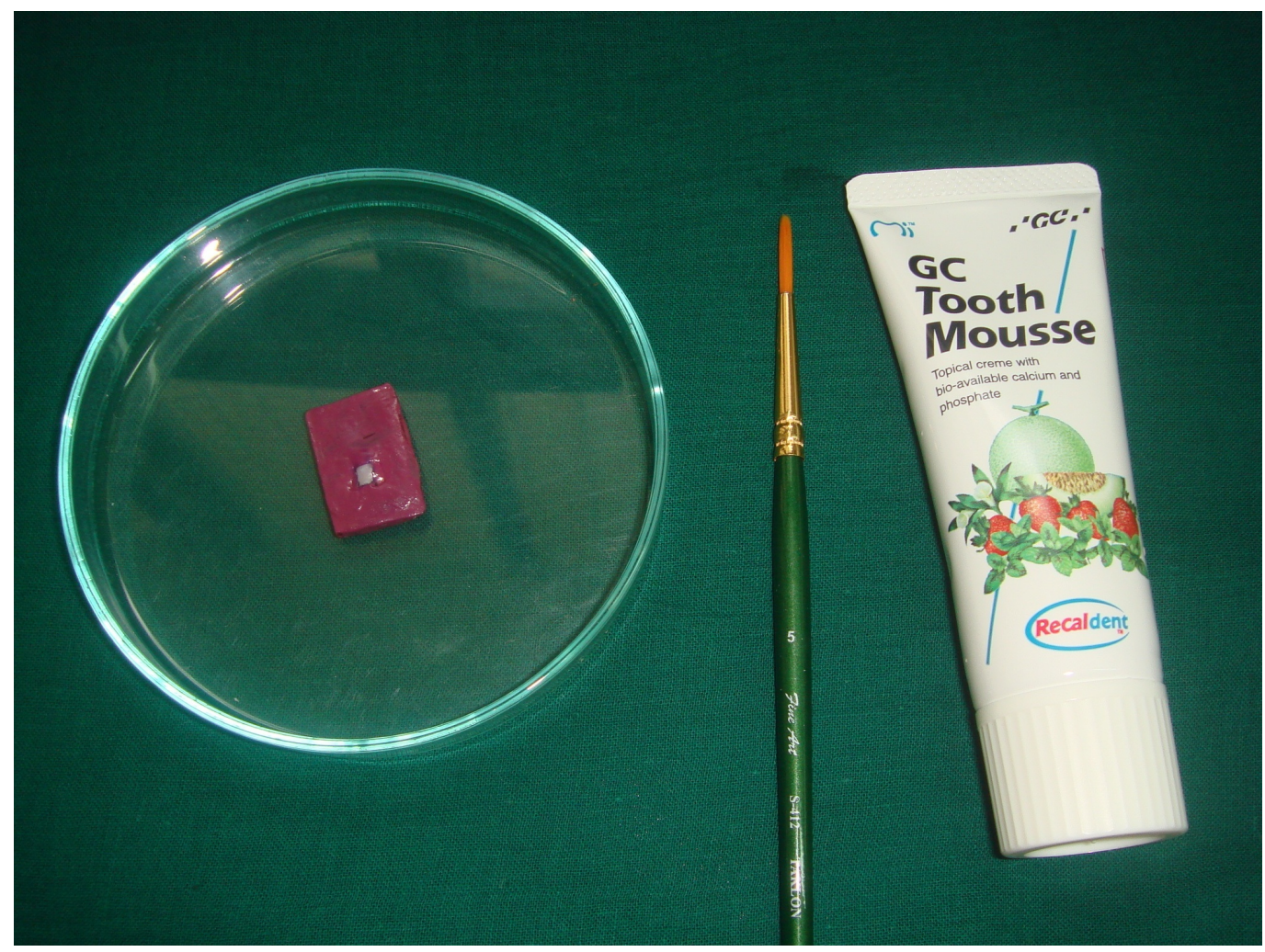

FIGURE 6: Group C: CPP-ACP treatment 


\section{Cureus}

The fourth quadrant was treated with calcium sodium phosphosilicate and was designated as group D (Figure 7).

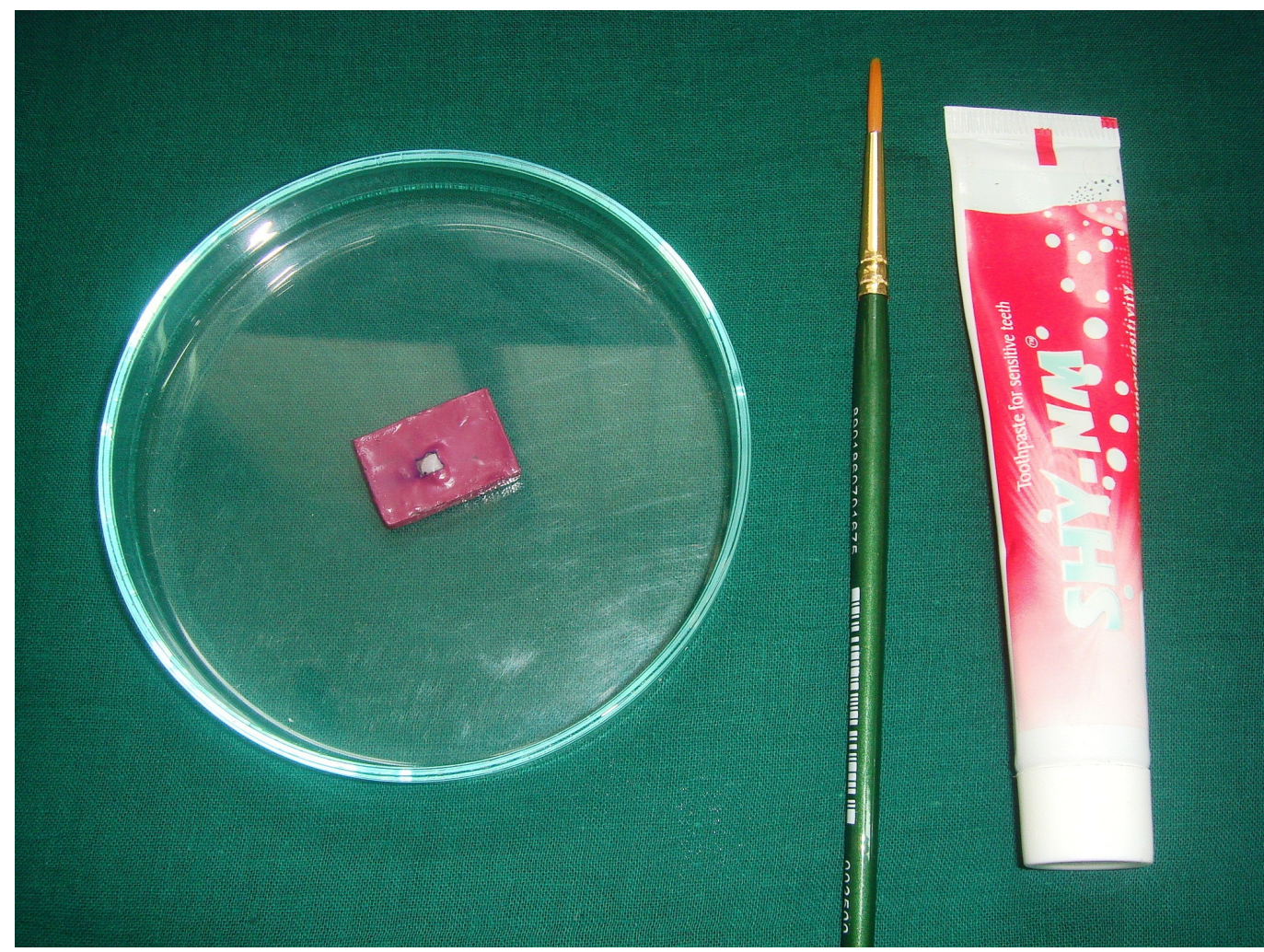

FIGURE 7: Group D: calcium sodium phosphosilicate (Novamin) treatment

The following protocol was followed [10]:

1) Applying the test materials on the specimens

2) Placing the specimens in the demineralizing solution for three hours

3) Washing the specimens in distilled water

4) Applying the test materials on the specimen

5) Washing the specimens in distilled water

6) Placing the specimens in synthetic saliva for 20 hours

\section{Cross-sectioning and staining}

The specimens were sectioned across the treatment window to expose the lesion depth (Figure 8). 


\section{Cureus}

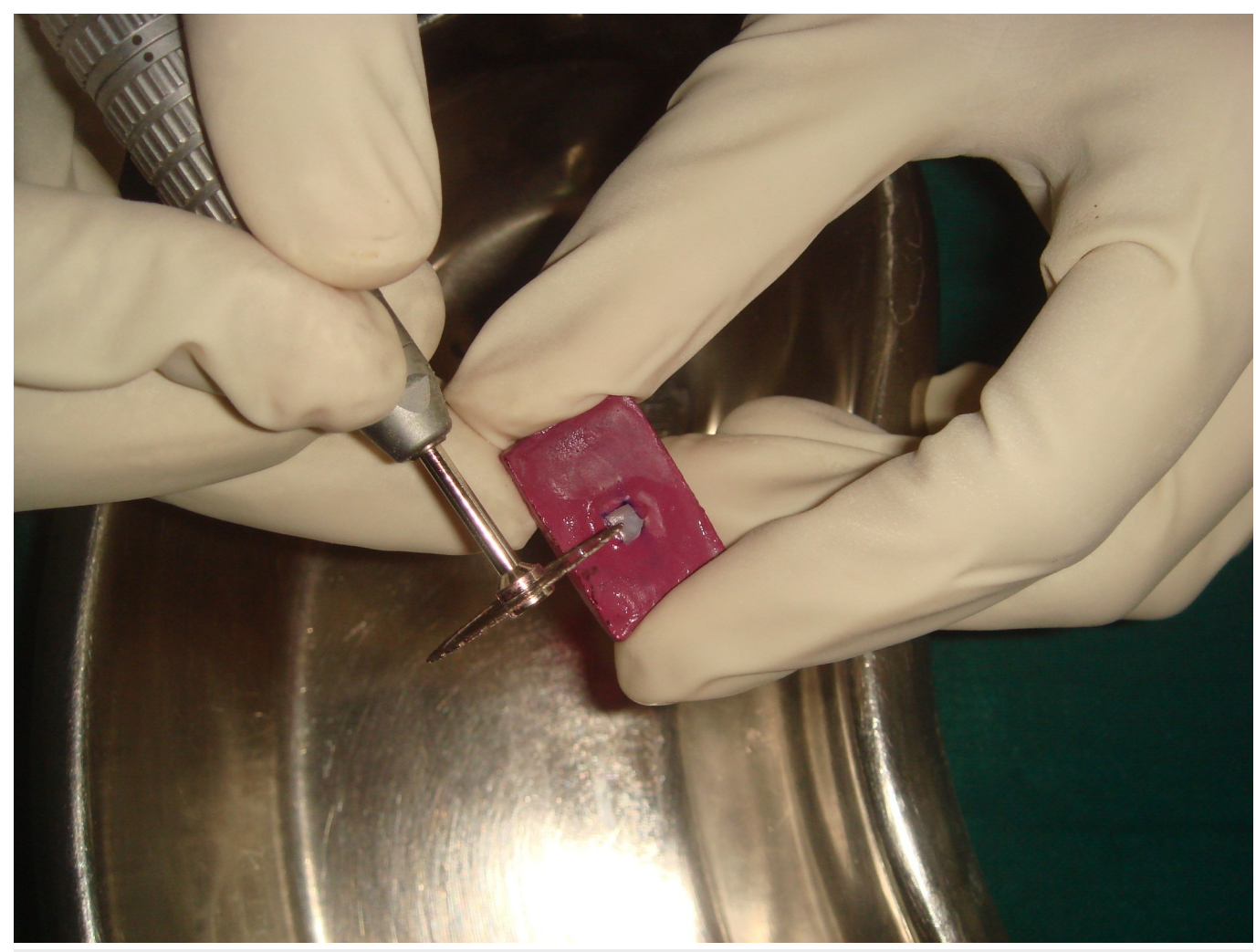

FIGURE 8: Cross-sectioning through the midline

Each section was stained by immersion in $0.1 \mathrm{mM}$ rhodamine solution for 24 hours [8].

\section{Confocal laser microscopic analysis}

The sections were subjected to a cross-sectional confocal laser microscopic analysis at the Department of Experimental Pathology, Sri Chitra Thirunal Institute of Medical Sciences. A Carl Zeiss Axiovert 200M inverted microscope (Göttingen, Germany) with a confocal attachment was used for this study (Figure 9). 


\section{Cureus}

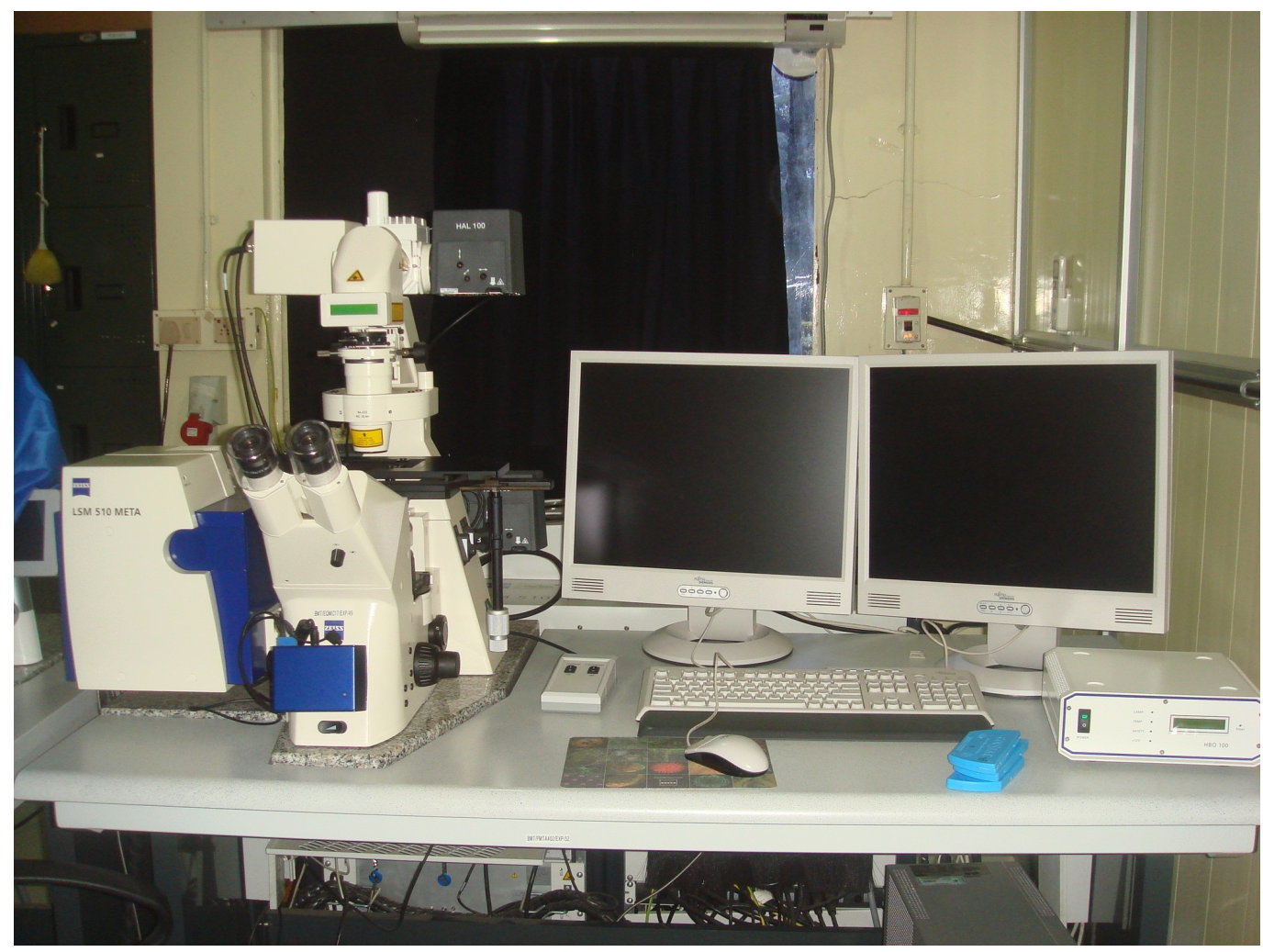

FIGURE 9: Confocal laser scanning microscope

This microscope used Carl Zeiss LSM 510 version 4 software (Göttingen, Germany) in order to visualize the images captured by the microscope on a computer screen. The cross-sectional analysis was based on digital images taken at controlled microscope settings. The area stained by the rhodamine dye appears red and is indicative of a demineralized area. Sound tooth and acrylic resin appear black, as they do not absorb any dye. Representative images are shown (Figure 10). 


\section{Cureus}

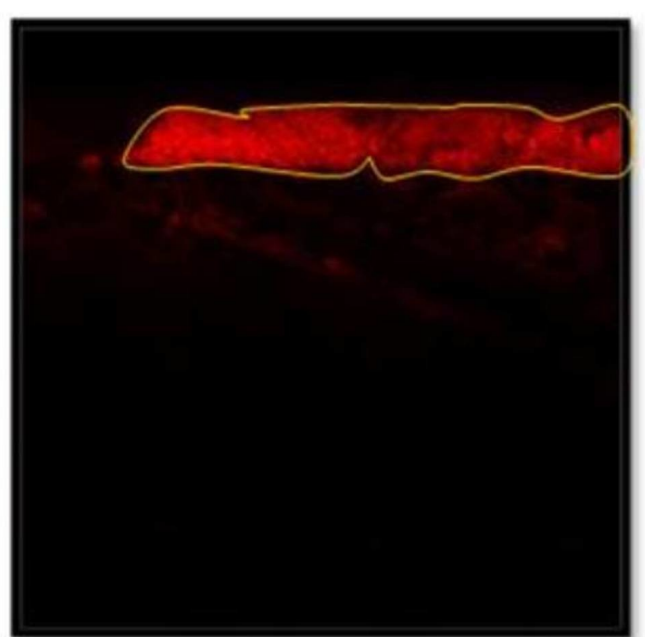

Group A

No Treatment

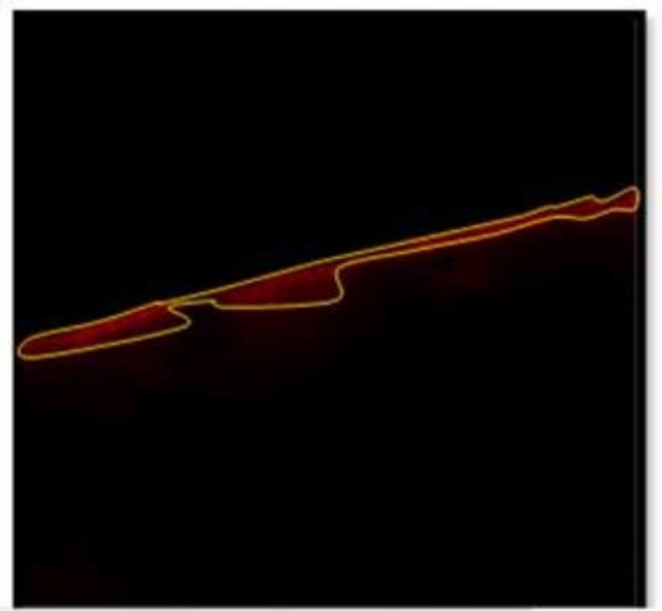

Group C

CPP-ACP

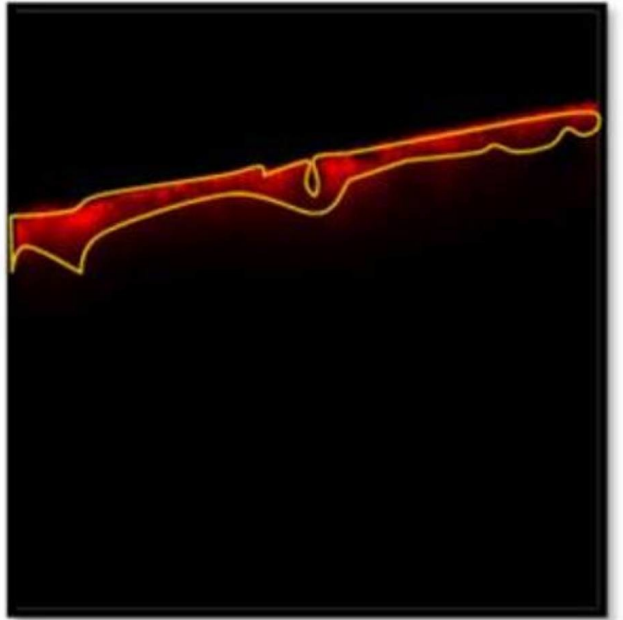

Group B

Monofluorophosphate

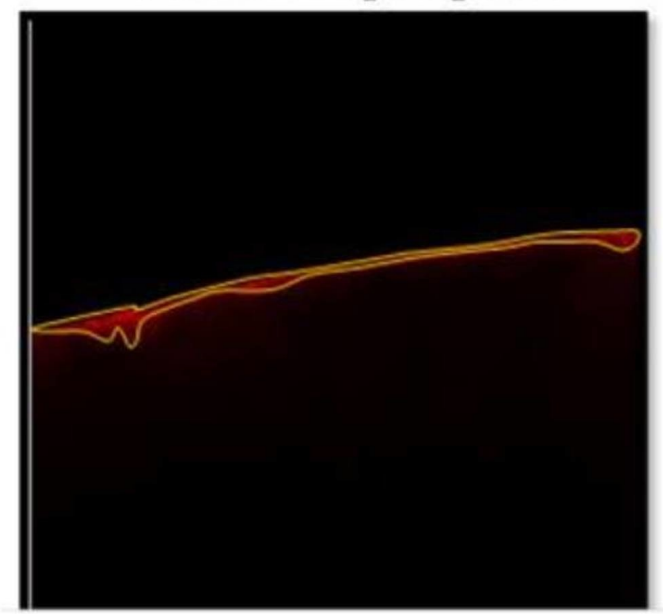

Group D

Calcium Sodium Phosphosilicate

\section{FIGURE 10: Representative images}

\section{Image analysis}

Image analysis was done using the imageJ software (National Institutes of Health, Maryland, US) and the following parameters were analyzed.

\section{Cross-sectional lesion area}

\section{Total fluorescence (total gray value)}

\section{Results}

The mean area and mean total fluorescence for the no treatment group were recorded as $16449.2 \mu 2$ and 759743.1, respectively. The group treated with monofluorophosphate dentifrice recorded the values as $73712 \mu 2$ and 233765.9. The values for the lesion area and total fluorescence for casein phosphopeptide-amorphous calcium phosphate group were $5776.6 \mu 2$ and 129470.8 respectively. The lowest values for lesion area and total fluorescence were 


\section{Cureus}

recorded by the calcium sodium phosphosilicate group as $3874.1 \mu 2$ and 107282.6, respectively. One-way ANOVA showed that the results were statistically significant $(p<0.01)$ (Tables $1-2)$.

\begin{tabular}{|l|l|l|l|}
\hline & & & \\
\hline \\
Group A No treatment & Mean value & SD & F \\
\hline Group B Monofluorophosphate & 16449.2 & 247.2 & 30 \\
Group C Casein Phosphopeptide-Amorphous Calcium Phosphate & 5776.6 & 264.5 & 30 \\
\hline Group D Calcium Sodium Phosphosilicate & 7371.3 & 113.1 & 30 \\
\hline
\end{tabular}

\section{TABLE 1: Comparison of cross-sectional demineralized lesion area based on group}

Actual $P$ value is $<2 e^{-16}$

\begin{tabular}{|c|c|c|c|c|c|}
\hline & Mean & SD & $\mathbf{N}$ & $\mathbf{F}$ & P value \\
\hline Group A No treatment & 759743.1 & 4847.4 & 30 & \multirow{4}{*}{$81616.44^{\star \star}$} & \multirow{4}{*}{0.000} \\
\hline Group B Monofluorophosphate & 233765.9 & 2168.6 & 30 & & \\
\hline Group C Casein Phosphopeptide-Amorphous Calcium Phosphate & 129470.8 & 4261.2 & 30 & & \\
\hline Group D Calcium sodium phosphosilicate & 107282.6 & 9577.2 & 30 & & \\
\hline
\end{tabular}

\section{TABLE 2: Comparison of total fluorescence (total gray value based on group}

Actual $P$ value is $<2 e-16$

Scheffe multiple comparisons proved that the variations in each group were also significant. As the lowest values were recorded by the calcium sodium phosphosilicate group, it emerged as the most effective remineralizing agent in this study. It was followed by CPP-ACP.

Monofluorophosphate was proved as the least effective remineralizing agent as the values recorded for lesion area and total fluorescence were the highest for this group.

\section{Discussion}

Fluoride is the most commonly used remineralizing agent. By acting on the dynamics of the caries process, fluoride is very effective in slowing down the progress of carious lesions. It not only inhibits demineralization but also aids in the remineralization of the enamel surface, leading to the formation of fluorohydroxyapatite or fluorapatite.

A potential problem with the mechanism of fluoride in remineralization is that for fluorapatite or fluorohydroxyapatite to form, calcium and phosphate ions are required, as well as fluoride ions. Several authors have now shown that enamel remineralization in situ and the retention of fluoride in plaque are dependent on the availability of calcium ions [11-12]. 
CPP-ACP and calcium sodium phosphosilicate are both based on calcium and phosphate ions. Calcium on the surfaces of the calcium and phosphate ion clusters primarily interacts with the casein phosphopeptides through the negatively charged residues of the peptides [3]. Casein phosphopeptides only weakly bind calcium and phosphate ions, thus allowing for a dynamic equilibrium between free and bound ions. This, therefore, provides a reservoir of bioavailable ions, which, on changes in the intraoral $\mathrm{pH}$, can be easily released to aid in the process of remineralization.

In aqueous environments, $\mathrm{Na}+$ particles in calcium sodium phosphosilicate immediately (within one minute) begin to exchange with hydrogen cations $(\mathrm{H}+$ or $\mathrm{H3O}+$ ) [13-15]. This rapid exchange of ions allows calcium ( $\mathrm{Ca} 2+)$ and phosphate (PO43-) species to be released from the particle structure. A modest, localized, transient increase in $\mathrm{pH}$ occurs that facilitates the precipitation of calcium and phosphate from the particles and from saliva to form a calcium phosphate (Ca-P) layer on tooth surfaces. As the reactions and the deposition of Ca-P complexes continue, this layer crystallizes into hydroxycarbonate apatite, which is chemically and structurally similar to biological apatite [13]. The results obtained showed that samples treated with calcium sodium phosphosilicate recorded the least values for the demineralized cross-sectional lesion area and total fluorescence. This suggests that very few demineralized areas were present in these samples. As the dye stains only demineralized areas, the area occupied by the dye was the least in these specimens, consequently, giving rise to the lowest values for the parameters measured. The values recorded for cross-sectional demineralized lesion area and total fluorescence were highest for the no treatment group, as they were not subjected to any surface treatment. This reinforces the fact that some form of dentifrice usage is a must for remineralization to occur and saliva alone is not adequate. The values recorded by the CPP-ACP group were higher than the calcium sodium phosphosilicate group, suggesting that the remineralization produced by the latter was more effective. Monofluorophosphate lead to minimal remineralization in this study.

Though casein phosphopeptide-amorphous calcium phosphate aids in subsurface remineralization, its use as-an-over the counter dentifrice has not been promoted. The cost of such products still remains high, and there is a need for their wider availability leading to increased usage and the benefits of the casein phosphopeptide-amorphous calcium phosphate technology. Another matter of concern is that plaque peptidases and phosphatases can degrade the phosphopeptides. The dephosphorylation of the phosphoserines of the Casein phosphopeptides by phosphatases substantially reduces the ability of the peptides to bind calcium and phosphate ions [16]. This leads to a loss of valuable calcium and phosphate ions and thus would hamper remineralization.

Calcium sodium phosphosilicate comes as a more economical alternative to CPP-ACP and according to this study, it is more effective than CPP-ACP. However, one consideration that must be kept in mind is that the remineralization with calcium sodium phosphosilicate leads to the formation of carbonate apatite while that with fluoride leads to the formation of fluorapatite. Fluorapatite is more resistant to further acid attack than carbonate apatite. The use of fluoride dentifrice can, therefore, be supplemented with calcium sodium phosphosilicate dentifrice for achieving better remineralization, as fluoride dentifrice is the most common and most economical dentifrice used in most households in India. Hence, measures to use a combination of calcium sodium phosphosilicate and fluoride must be explored.

This study was an in vitro study. In vitro models are mechanistically limited in three key ways: an inadequate simulation of the biological aspects of caries, a difficulty in matching solid/solution ratios occurring in vivo, and artifacts associated with substrate choice/reaction conditions 
To obtain meaningful data, in vitro analysis requires the investigator to eliminate as much experimental variability as possible. The relative success of this study should be credited to meticulous standardization in each procedural step. Sample preparations, solutions, and processes were all subject to strict uniformity control for all tooth sections. This leaves only inherent specimen variability unaccounted.

Therefore, within the limits of the study, the results of using calcium sodium phosphosilicate as a remineralizing agent seem promising. Further clinical trials are, however, necessary to make public recommendations.

\section{Conclusions}

The results of this study suggest that among the three agents tested, calcium sodium phosphosilicate is the most effective remineralizing agent followed by casein phosphopeptideamorphous calcium phosphate. Though monofluorophosphate is effective as a remineralizing agent, its effectiveness is not as good as the other two agents when tested under the conditions mentioned in this study.

\section{Additional Information \\ Disclosures}

Human subjects: All authors have confirmed that this study did not involve human participants or tissue. Animal subjects: All authors have confirmed that this study did not involve animal subjects or tissue. Conflicts of interest: In compliance with the ICMJE uniform disclosure form, all authors declare the following: Payment/services info: All authors have declared that no financial support was received from any organization for the submitted work. Financial relationships: All authors have declared that they have no financial relationships at present or within the previous three years with any organizations that might have an interest in the submitted work. Other relationships: All authors have declared that there are no other relationships or activities that could appear to have influenced the submitted work.

\section{References}

1. Featherstone JD: The science and practice of caries prevention . J Am Dent. 2000, 131:887-899.

2. ten Cate JM, Exterkate RAM, Buijs MJ: The relative efficacy of fluoride toothpastes assessed with pH cycling. Caries Res. 2006, 40:136-141. 10.1159/000091060

3. Cross KJ, Huq NL, Palamara JE, Perich JW, Reynolds EC: Physicochemical characterization of casein phosphopeptide-amorphous calcium phosphate nanocomplexes. J Biol Chem. 2005, 280:15362-15369.

4. Wefel JS: NovaMin: likely clinical success . Adv Den Res. 2009, 21:40-43. 10.1177/0895937409335622

5. Pantera EA, Schuster GS: Sterilization of extracted human teeth . J Dent Educ. 1990, 54:283285.

6. Richards A, Fejerskov O, Baelum V: Enamel fluoride in relation to severity of human dental fluorosis. Adv Dent Res. 1989, 3:147-153. 10.1177/08959374890030021301

7. Kumar VLN, Itthagarun A, King NM: The effect of casein phosphopeptide-amorphous calcium phosphate on remineralization of artificial caries-like lesions: an in vitro study. Aust Dent J. 2008, 53:34-40. 10.1111/j.1834-7819.2007.00006.x

8. Evaluation of novamin as an adjunct to fluoride for caries remineralization. Novamin Research Report. (2004). Accessed: June 23, 2018: http://www.ufdc.ufl.edu/ufe0007162/00001.

9. Sato Y, Sato T, Niwa M, Aoki H: Precipitation of octa calcium phosphates on artificial enamel in artificial saliva. J Mat Sci. 2006, 17:1173-1177. 10.1007/s10856-006-0545-4

10. Argenta RMO, Tabchoury CPM, Cury JA: A modified pH-cycling model to evaluate fluoride effect on enamel demineralization [Article in Portueguese]. Pesqui Odontol Bras. 2003, 17:241-246. 10.1590/S1517-74912003000300008 


\section{Cureus}

11. Reynolds EC, Cai F, Cochrane NJ, Shen P, Walker GD, Morgan MV, Reynolds C: Fluoride and casein phosphopeptide-amorphous calcium phosphate. J Dent Res. 2008, 87:344-348. $10.1177 / 154405910808700420$

12. Vogel GL, Schumacher GE, Chow LC, Takagi S, Carey CM: Ca pre-rinse greatly increases plaque and plaque fluid F. J Dent Res. 2008, 87:466-469. 10.1177\%2F154405910808700513

13. Anderrson $\mathrm{OH}$, Kangasniemi I: Calcium phosphate formation at the surface of bioactive glass in vitro. J Biomed Mater Res. 1991, 25:1019-1030. 10.1002/jbm.820250808

14. Hench LL, Andersson O: Introduction to Bioceramics: Bioactive Glasses. World Scientific. L Hench, J Wilson (ed): World Scientific, Singapore; 1993. 41-62. 10.1142/9789814317351_0003

15. Cerruti MG, Greenspan D, Powers K: An analytical model for the dissolution of various particle size samples of bioglass in TRIS buffered solution. Biomaterials. 2005, 26:4903-4911. 10.1016/j.biomaterials.2005.01.013

16. Cochrane NJ, Saranathan S, Cai F, Cross KJ, Reynolds EC: Enamel subsurface lesion remineralisation with casein phosphopeptide stabilised solutions of calcium, phosphate and fluoride. Caries Res. 2008, 42:88-97. 10.1159/000113161 